\title{
Both younger and elderly patients in pain are willing to undergo knee replacement despite the COVID-19 pandemic: a study on surgical waiting lists
}

\author{
Enrique Gómez-Barrena ${ }^{1}$ [ - Israel Rubio-Saez ${ }^{1} \cdot$ Norma G. Padilla-Eguiluz $^{1} \cdot$ Pablo Hernandez-Esteban $^{1}$
}

Received: 24 March 2021 / Accepted: 10 May 2021 / Published online: 20 May 2021

(c) European Society of Sports Traumatology, Knee Surgery, Arthroscopy (ESSKA) 2021

\begin{abstract}
Purpose To identify factors influencing patient's availability to re-schedule primary total knee replacement (TKR) or revision (RKR) surgery after the lockdown (March-May 2020) during the COVID-19 pandemic.

Methods A prospective cohort study through a telephone survey was performed in 156 patients (143 for primary and 13 for revision) included in the TKR and RKR surgical waiting list before March 2020. Contact of each patient with COVID-19, stress and anxiety, perceived pain, and function were obtained in the interviews, and also the preference of each patient to have re-scheduled surgery (early or late). Finally, we registered their response (acceptance or refusal) when surgery was effectively re-scheduled.

Results 88 out of 156 patients waiting for knee replacement (76/143 of those waiting for TKR, 12/13 of those waiting for RKR) declared themselves ready for surgery in less than 1 month. When re-scheduled, 115 patients underwent surgery and 41 refused. Significantly different preferences were found for age (more prone to surgery if under 65), revision surgery (more readily available), pain ( $7.9 \pm 1.7 / 10$ in NRS in those undergoing surgery, 5.6 $\pm 2.3 / 10$ in those refusing, $p=0.000$ ), or COVID-19 diagnosis, but not other close contact with COVID-19, comorbidities, stress, or anxiety. A logistic regression model confirmed that revision surgery (OR 9.33), perceived severe pain (OR 5.21), and age under 65 years (OR 5.82) were significantly associated with patient preference. The probability of patients over 65 to prefer early surgery reached $60 \%$ only with pain at or above $9 / 10$.

Conclusions Surgical timing preferences for knee replacement vary between patients older than 65 years (immediate surgery only when pain is intense) and younger patients (immediate surgery no matter the amount of pain). Even if COVID-19 severely stroke our population, the need for knee replacement stood in the young population and even in the aged population at risk for COVID when pain was important.
\end{abstract}

Keywords COVID-19 $\cdot$ Knee replacement $\cdot$ Patient availability $\cdot$ Waiting list $\cdot$ Surgery preferences

\section{Introduction}

The necessity of knee replacement is increasing due to the demography and the demands on the associated quality of life and function. Consequently, the waiting time to perform the procedure [4], particularly when resources are limited or blocked, may be unduly extended. This situation is a

Enrique Gómez-Barrena

egomezb@salud.madrid.org; enrique.gomezbarrena@uam.es

1 Orthopaedic Surgery and Traumatology Department, Knee Unit, Hospital La Paz, Universidad Autónoma de Madrid, Po Castellana 261, 28046 Madrid, Spain matter of concern as it is proven that waiting time longer than 6 months can be associated with worse outcomes [8].

In addition, the functional status declines progressively during the waiting time [2] and some patients may deteriorate even from severe conditions [21]. Prioritization systems have been proposed to classify patients and select who should undergo earlier surgery $[7,10]$.

COVID-19 pandemics spread all around the world on a first wave, but with a significant impact in Spain, and mainly in the region of Madrid, March 2020. Our hospital was blocked while treating one of the largest cohorts reported in Europe. [5] Adopted measures included a full surgical lockdown for elective procedures such as knee replacement, with exception of related acute complications such as infection or 
fracture, to increase the availability of intensive care beds for COVID-19 patients. Those measures lead to increasing the waiting time and the number of patients for knee replacement, not receiving the appropriate care in the context of a pandemic that negatively affected physical and mental health, particularly in elderly population [26]. High rates of anxiety, insomnia, depression, and other mental health disorders have been reported in relationship with the lockdown and COVID-19 pandemic [19].

After the first wave of the COVID-19 pandemic seriously affected elective joint replacement surgical activity in many European countries [24], the surgical re-opening faced different patient attitudes towards re-scheduling their surgeries, in view that the pandemic is not fully over, and the situation is still uncertain. Of course, the re-opening has had significant ethical and economical implications [15]. Therefore, national and local guidance was developed and protocols put in place in the patient pathway for primary arthroplasty to allow for a safe return [14]. According to the European Knee Associates (EKA) and the European Hip Society (EHS), some recommendations for resuming elective surgery [13] in the setting of the SARS-COV-2 pandemic have been established in a good-to-excellent agreement.

In this context, even if patients in the waiting list may be reluctant to undergo surgery after the 1st wave of the pandemic, our hypothesis was that young patients, but also many elderly patients, would be willing to undergo knee replacement surgery despite the pandemic situation and the related risks.

The aim of this study was to identify factors influencing the patient preference to re-schedule primary total knee replacement (TKR) or revision (RKR) surgery after the lockdown occurred during the first wave of the COVID-19 pandemic. As a secondary objective, the study aimed to explore the cause why knee replacement surgery would be finally performed or refused by patients in the waiting list prior to the pandemic.

\section{Patients and methods}

This is a prospective, observational, cohort study of patients from the waiting list for TKR or RKR surgery. Patients were included before the suspension of the elective activity due to the 1st wave of the COVID-19 pandemic (March-June 2020) in the Orthopaedic Surgery Department of La Paz University Hospital, Madrid, Spain.

\section{Population}

Eligibility criteria included patients over 18 years old, in the surgical waiting list for knee replacement (whether TKR or RKR), able to give verbal consent to participate in the study, as approved by the Ethical Committee of La Paz University Hospital (Code: PI-4298, 31 July 2020). Patients were not included if surgery was denied after anaesthesiology consultation or if patients required associated techniques other than TKR or RKR. A total of 163 patients were identified in the suspended surgical waiting list, 150 TKR and 13 RKR. Seven patients were excluded ( 5 decline their participation, 1 was dead during the 1 st wave of the pandemic, and 1 left the country) and 156 patients were included in the study. Of these, 17 showed difficulties to answer the stress and anxiety survey on the telephone or refused to answer these questions.

\section{Variables}

Data were obtained from the clinical history of each patient and through a telephone survey after the first COVID-19 wave.

Variables obtained from the clinical history included age, sex, Body Mass Index (BMI), American Society of Anaesthesiologists physical status category (ASA), comorbidities, and risk factors that could compromise their survivorship in case of COVID-19 infection, identified by the Center for Disease Control and Prevention (CDC)[16]: asthma, chronic pulmonary disease, chronic cardiac disease, chronic renal disease, severe obesity, immunocompromised disease, and hepatic disease. These CDC risk factors were categorized, according to the British Orthopaedic Association, as low risk ( $<65$ years without other risk factors), medium risk ( $\geq 65$ years without other risk factor or $<65$ years with another risk factor), high risk ( $\geq 65$ years with another risk factor or $\leq 65$ years with two more risk factors), or very high risk (all patients with more than three risk factors) [6].

Variables obtained through the telephone interview included the patient's perceived pain through the Numeric Rating scale (NRS) from 0 to 10 points (where 0 means no pain at all and 10 means the worse pain ever felt) [11, 12]. The perceived patient capacity to perform normal activity was subjectively quantified by the patient through a proposed scale of functional limitation from 0 to 10 points (where 0 means normal functionality and 10 means completed limitation). The wandering capability was evaluated using the Functional Ambulation Capacity scale (FAC) from 0 to 5 [25]. Contact with COVID-19 for each patient was evaluated thorough a clinical-epidemiological questionnaire launched by the Ministry of Health in XXXX, defining if the patient has undergone COVID-19 infection or compatible symptoms and if the patient has had close contact with a positive case at home or at work [22]. Stress and anxiety were assessed through the Perceived Stress Scale (PSS) and the Inventory of Situations and Responses of Anxiety (ISRA), as recommended by the XXXX Ministry of Health during the pandemic.[20] Stress in the previous month was considered high for a score of 23 or higher, and very high if 
32 points or higher were reached. Anxiety was considered acceptable if the score was less than 16 points, in men, or less than 19 in women. Men scoring 16 or more, and women with 19 or more points were placed in the 75 th centile or higher (more anxiety than $75 \%$ of the same sex population). Patients exceeding 30 points reached the 99th centile (more anxiety than $99 \%$ of the same sex population).

Finally, patients were asked about their preference to re-schedule surgery in less than 1 month, 1-3 months, 3-6 months, or more than 6 months, and this was used as a dependent variable. This variable was dichotomized in patients who preferred to schedule early surgery (including the $<1$ month and 1-3 month options) and patients who preferred to postpone (including 3-6 months and $>6$ months options).

\section{Follow-up}

The surgical activity was resumed during the summer of 2020. Clinical charts were also reviewed in February of 2021 to confirm if patients effectively underwent surgery when scheduled, or else if they refused surgery.

\section{Analysis}

Data were compared between the dependent variable (preference to early re-scheduling or to postponing for a late surgery) using descriptive and analytical statistical methods, including mean, standard deviation of the mean, frequency, and percentages. To identify differences between groups, $t$ test, Mann-Whitney test, Pearson's Chi-square test, or Fisher exact test (if data contained less than 5 cases per cell) were used, as deemed appropriate, at a confidence level of $95 \%$. Therefore, $p$ values $<0.05$ were considered significant.

Multivariate logistic regression was modelled using the preference to re-schedule surgery (early vs. late) as the dependent variable, and the type of surgery (TKR or RKR), age category ( $<65$ years, $>65$ years), ASA category (I-II, III), perceived pain (mild-to-medium pain up to 6 points vs severe pain between 7 and 10 points in NRS), and confirmed COVID-19 past infection (yes/no) as independent variables, identified through logistic bivariate models. The odds ratio was considered statistically significant if the $95 \%$ interval did not cross 0 .

Using the follow-up data, patient characteristics and variables from questionnaires were also compared between those who underwent surgery and those who refused it. A second logistic regression was similarly modelled including the outcome (yes/no surgery) as the dependent variable. Finally, the predicted probability of both logistic models was generated and compared using the $t$ test for paired data. The statistical analysis was conducted with Stata software v.12 (StataCorp.
2019. Stata Statistical Software: Release 16. College Station, TX: StataCorp LLC).

\section{Results}

The mean age of the participants was $74.9 \pm 8.3$ years (Min: 48; Max: 90), 78\% were females, the mean BMI was $30.6 \pm 4.9$ (Min: 21; Max: 49), and according to the ASA physical status, $6 \%$ were classified as ASA I $(n=9), 55 \%$ as ASA II $(n=86)$, and $39 \%$ as ASA III $(n=61)$. The mean perceived pain was $7 \pm 2$ over 10 points in the NRS, and $89 \%$ of the patients could walk, as evaluated through the FAC scale ( $4.5 \pm 0.8)$, although the subjective perception of functional limitation was $6 \pm 2$ over 10 points. The mean PSS value was $13.34 \pm 8.8$ with mean punctuation in females of $13.9 \pm 8.7$ and $10.8 \pm 9.2$ in men. Globally, $84 \%(n=117)$ of the patients recorded an acceptable level of stress, $11 \%$ $(n=15)$ a high level, and 5\% $(n=7)$ a very high level of stress. Although raw differences by sex were statistically significant (Mann-Whitney test, $p=0.0184$ ), no gender differences were observed in the categorical variable of stress (Fisher's exact test; $p=0.566$, n.s.). Regarding anxiety, the mean punctuation of the ISRA index was $9.5 \pm 6.5$ in females and $6.6 \pm 6.7$ in males. Globally, $88 \%(n=124)$ laid at an acceptable level, while $11 \%(n=16)$ reported higher anxiety than $75 \%$ of the population. Again, no gender differences were observed in the categorical variable (Fisher's exact test; $p=0.598$, n.s.).

Twenty-five percent $(n=39)$ of our patients reported close contact with COVID-19 during the 1st COVID-19 wave. Data from the COVID-19 epidemiological survey (multiple answers were possible) included 7\% $(n=11)$ who received a COVID-19 diagnosis, $12 \%(n=19)$ who reported to live or work with somebody infected, $2 \%(n=3)$ who lived in a nursing home where COVID-19 was diagnosed in other residents, and 14\% $(n=22)$ who had suffered from COVID19 compatible symptoms without COVID-19 confirmatory diagnosis (including cough $64 \%$, fever $55 \%$, asthenia $45 \%$, ageusia $30 \%$, anosmia $24 \%$, dyspnoea $15 \%$, vomiting $15 \%$, odynophagia $12 \%$, headache $6 \%$, myalgia $3 \%$, conjunctivitis $3 \%$, and rhinorrhoea $2 \%$ ). Regarding the COVID-19-related risk, 144 patients $(92 \%)$ presented with risk factors that could compromise their survival in case of COVID-19 infection, according to the $\mathrm{CDC}$. Using the BOA risk category scale, $7 \%$ were allocated to low risk $(n=12), 23 \%$ to moderate risk $(n=37), 41 \%$ to high risk $(n=64)$, and $28 \%$ to very high risk $(n=44)$.

\section{Patient preferences}

Patients in the TKR waiting list who preferred to schedule their surgery early showed higher perceived pain and higher 
perceived functional limitation. Patients preferring primary TKR early surgery (76 out of $143,53 \%$ ) included less proportion of patients aged over 65 years than in the group of patients who preferred to postpone surgery (Table 1). Comparing TKR and RKR waiting list, we found that, on average, patients in the RKR waiting list were older, classified with higher ASA, with more difficulty to ambulate (FAC) than patients waiting for TKR (Table 2), but 12 out of 13 (92\%) preferred to have surgery as early as possible.

When assessing COVID-19-related risk factors, only age above 65 proved statistically significant differences $(p=0.002)$ between primary TKR patients preferring early surgery and those preferring late, postponed surgery (Supplement, Table S1). Between primary TKR and RKR patients, stress was apparently higher in primary TKR patients, but with a high rate of non-respondents (Supplement, Table S2).

A total of 156 observations were included in the logistic regression model, to test patients' preference about early or late surgery (Table 3). Patients were 9 times at more odds to schedule their surgery if waiting for RKR than for TKR; patients younger than 65 were almost 6 times more likely to prefer early scheduling their surgery than older patients, and patients with severe perceived pain (equal or over 7 points) were 5 times more likely to schedule their surgery than patients with less pain (Table 3).

\section{Final outcome.}

Of the 88 patients who showed preference for early scheduling their surgery when surveyed, $78(89 \%)$ accepted to undergo surgery when scheduled, and $10(11 \%)$ refused, due to personal choice $(n=7)$, surgery contraindication $(n=2)$, or clinical improvement $(n=1)$. Of the 68 patients who preferred to delay their surgery when surveyed, 37 (55\%) still accepted to undergo surgery when scheduled, while $31(45 \%)$ refused it, due to personal choice $(n=28)$, other reason $(n=2)$, and surgery contraindication $(n=1)$.
Table 1 Comparison of patients in the knee replacement waiting list (primary TKR), by preference to schedule early or late surgery after the end of surgical lockdown

\begin{tabular}{llll}
\hline Variables & $\begin{array}{l}\text { Preferred early surgery } \\
(n=76) \\
\text { Mean } \pm \mathrm{SD}, n(\%)\end{array}$ & $\begin{array}{l}\text { Preferred late, postponed } \\
\text { surgery }(n=67) \\
\text { Mean } \pm \mathrm{SD}, n(\%)\end{array}$ & $P$ value \\
\hline Age (years) & $73.1 \pm 9.5$ & $75.7 \pm 6.6$ & $0.090^{* *}$ \\
Sex (females) & $58(52 \%)$ & $54(48 \%)$ & $0.535^{* * *}$ \\
BMI & $30.7 \pm 4.5$ & $29.8 \pm 4.5$ & $0.251^{* *}$ \\
ASA & $2.3 \pm 0.5$ & $2.3 \pm 0.6$ & $0.878^{*}$ \\
I-II & $49(54 \%)$ & $41(46 \%)$ & $0.772^{* * *}$ \\
III & $27(52 \%)$ & $25(48 \%)$ & $0.000^{*}$ \\
Perceived pain (NRS) & $7.9 \pm 1.7$ & $5.6 \pm 2.3$ & $0.000^{*}$ \\
Perceived functional limitation & $5.5 \pm 2.1$ & $3.9 \pm 2$ & $0.212^{* *}$ \\
FAC punctuation & $4.5 \pm 0.8$ & $4.7 \pm 0.6$ & \\
\hline
\end{tabular}

$S D$ standard deviation; $B M I$ body mass index; NRS numeric rating scale; $F A C$ functional ambulation capacity

*t test; **Mann-Whitney test; ***Pearson's Chi-square test

\begin{tabular}{llll}
\hline Variables & $\begin{array}{l}\text { TKR surgery }(n=76) \\
\text { Mean } \pm \text { SD, } n(\%)\end{array}$ & $\begin{array}{l}\text { RKR surgery } \\
(n=12) \\
\text { Mean } \pm \mathrm{SD}, n(\%)\end{array}$ & $P$ value \\
\hline Age (years) & $73.1 \pm 9.5$ & $80 \pm 5.4$ & $0.000^{*}$ \\
Sex (females) & $58(76 \%)$ & $9(75 \%)$ & $0.921^{* * *}$ \\
BMI & $30.7 \pm 4.5$ & $33.4 \pm 7.2$ & $0.179^{* *}$ \\
ASA & $2.3 \pm 0.5$ & $2.7 \pm 0.5$ & $0.037^{* *}$ \\
I-II & $49(65 \%)$ & $4(33 \%)$ & $0.057^{* * *}$ \\
III & $27(36 \%)$ & $8(67 \%)$ & \\
Perceived pain (NRS) & $7.9 \pm 1.7$ & $8.5 \pm 1.5$ & $0.236^{*}$ \\
Perceived functional limitation & $5.5 \pm 2.1$ & $7.6 \pm 1.8$ & $0.079 *$ \\
FAC punctuation & $4.5 \pm 0.8$ & $3.9 \pm 1.3$ & $0.032^{* *}$ \\
\hline
\end{tabular}

$T K R$ total knee replacement; $R K R$ revision knee replacement; $S D$ standard deviation; $B M I$ body mass index; NRS numeric rating scale; FAC functional ambulation capacity

*t test; **Mann-Whitney test; ***Pearson's Chi-square test 
Table 3 Variables associated with the patient preference for early scheduling (less than 3 months) of their knee replacement surgery (TKR/ $\mathrm{RKR})$, at the end of the COVID-19 lockdown, versus the preference to postpone the surgery

\begin{tabular}{|c|c|c|c|c|}
\hline Variables & Odds ratio & Low CI, 95\% & High CI, 95\% & $P$ value \\
\hline \multicolumn{5}{|l|}{ Type of surgery } \\
\hline TKR & 1 & & & \\
\hline RKR & 9.33 & 1.09 & 79.80 & 0.041 \\
\hline \multicolumn{5}{|l|}{ Age category } \\
\hline Over or equal to 65 years & 1 & & & \\
\hline Lower than 65 years & 5.82 & 1.47 & 23.07 & 0.012 \\
\hline \multicolumn{5}{|l|}{ ASA } \\
\hline $\mathrm{I}-\mathrm{II}$ & 1 & & & \\
\hline III & 1.22 & 0.56 & 2.65 & 0.607 \\
\hline \multicolumn{5}{|c|}{ Perceived pain (NRS) category } \\
\hline $0-6$ points & 1 & & & \\
\hline $7-10$ points & 5.21 & 2.51 & 10.86 & 0.000 \\
\hline \multicolumn{5}{|c|}{ COVID-19 epidemiological survey: confirmed past infection } \\
\hline No & 1 & & & \\
\hline Yes & 1.07 & 0.22 & 5.21 & 0.931 \\
\hline _cons & 0.29 & 0.04 & 2.05 & 0.220 \\
\hline
\end{tabular}

$T K R$ total knee replacement; $R K R$ revision knee replacement; $N R S$ numeric rating scale Model adjustment: $\operatorname{LR} \chi^{2}(5)=42.11 ; p=0.000$; Pseudo $R^{2}=0.1986 ; n=156$
This comparison was statistically significant (Pearson's Chi-square test; $p=0.000)$. Seventy-four percent $(n=106)$ of patients waiting for TKR effectively underwent surgery, and so did 69\% $(n=9)$ of RKR patients (Fisher's exact test; $p=0.460$, n.s.).

The age, perceived pain, perceived functional limitation, ASA grade, CDC risk factors, and history of COVID-19 significantly differed in those patients who finally agreed to schedule surgery and those who refused. We found that $100 \%(n=21)$ of patients younger than 65 years and $70 \%(n=94)$ of patients aged 65 years or more decided to undergo surgery (Fisher's exact test; $p=0.001$ ). The mean perceived pain in patients who underwent surgery was $7.3 \pm 2$, while pain was $5.9 \pm 2.5$ in patients who refused surgery (Mann-Mann-Whitney test; $p=0.003$ ); the mean perceived functional limitation in patients who underwent surgery was $6.4 \pm 1.9$, and $5.4 \pm 2.1$ in patients who refused surgery (Mann-Mann-Whitney test; $p=0.005$ ). Eighty-two per cent $(n=77)$ of patients with ASA classification lower than III, and $62 \%(n=38)$ of patients with ASA classification of III decided to undergo surgery (Person Chi-square test; $p=0.006$ ), as seen in Table 4 .

Regarding the influence of risk factors related to COVID19 , the presence of CDC risk factors influenced the acceptance of surgery $(71 \%, n=102$, patients with CDC risk factors and $100 \%, n=13$, patients without CDC risk factors finally accepted their surgery, Fisher's exact test; $p=0.021$ ). But most importantly, patients having received a COVID19 diagnosis were significantly less prone to accept surgery. Forty-five percent $(n=5)$ of patients having received a diagnosis of COVID-19 infection, and 76\% $(n=110)$ of
Table 4 Comparison of patients from the knee replacement waiting list who effectively underwent knee replacement surgery (TKR/ RKR), at the end of the COVID-19 lockdown, versus those who refused it

\begin{tabular}{|c|c|c|c|}
\hline Variables & $\begin{array}{l}\text { Underwent } \\
\text { surgery } \\
(n=115) \\
\text { Mean } \pm \text { SD, } \\
n(\%)\end{array}$ & $\begin{array}{l}\text { Refused } \\
\text { surgery } \\
(n=41) \\
\text { Mean } \pm \mathrm{SD}, \\
n(\%)\end{array}$ & $P$ value \\
\hline \multicolumn{4}{|l|}{ Type of surgery } \\
\hline TKR & $106(74 \%)$ & $37(25 \%)$ & $0.745 * * *$ \\
\hline RKR & $9(69 \%)$ & $4(31 \%)$ & \\
\hline Age (years) & $73.7 \pm 8.8$ & $78.1 \pm 5.4$ & $0.003^{*}$ \\
\hline \multicolumn{4}{|l|}{ Age category } \\
\hline $\begin{array}{l}\text { Older or equal to } \\
65 \text { years }\end{array}$ & $94(70 \%)$ & $41(30 \%)$ & $0.001 * * *$ \\
\hline Younger than 65 years & $21(100 \%)$ & 0 & \\
\hline \multicolumn{4}{|l|}{ Sex } \\
\hline Female & $87(71 \%)$ & $35(29 \%)$ & $0.196 * * *$ \\
\hline Male & $28(82 \%)$ & $6(18 \%)$ & \\
\hline \multicolumn{4}{|l|}{ ASA category } \\
\hline I-II & $77(82 \%)$ & $17(18 \%)$ & $0.006 * * *$ \\
\hline III & $38(62 \%)$ & $23(38 \%)$ & \\
\hline Perceived pain (NRS) & $7.3 \pm 2.0$ & $5.9 \pm 2.5$ & $0.003 * *$ \\
\hline $\begin{array}{l}\text { Perceived functional } \\
\text { limitation }\end{array}$ & $6.4 \pm 1.9$ & $5.4 \pm 2.1$ & $0.005^{* *}$ \\
\hline FAC punctuation & $4.56 \pm 0.80$ & $4.58 \pm 0.89$ & $0.692 * *$ \\
\hline
\end{tabular}

$S D$ standard deviation; NRS: numeric rating scale

$* t$ test; **Mann-Whitney test; ***Pearson Chi-square test or Fisher's exact test if less than 5 cases per cell 
patients with no history of COVID-19 finally underwent surgery (Fisher's exact test; $p=0.037$ ). Further description of COVID-19 related risk factors can be seen in Table S3 (Supplement).

A total of 156 observations were included in the logistic regression model, to test the probability to effectively

Table 5 Variables associated with patients from the knee replacement waiting list who effectively underwent their knee replacement surgery (TKR/RKR) at the end of the COVID-19 lockdown, versus those who refused it

\begin{tabular}{|c|c|c|c|c|}
\hline Variables & Odds ratio & Low CI, 95\% & High CI, 95\% & $P$ value \\
\hline \multicolumn{5}{|c|}{ Type of surgery } \\
\hline TKR & 1 & & & \\
\hline RKR & 1.13 & 0.29 & 4.44 & 0.856 \\
\hline Age & 0.94 & 0.89 & 0.99 & 0.046 \\
\hline \multicolumn{5}{|l|}{ ASA } \\
\hline I-II & 1 & & & \\
\hline III & 0.45 & 0.19 & 1.05 & 0.066 \\
\hline \multicolumn{5}{|c|}{ Perceived pain (NRS) category } \\
\hline $0-6$ points & 1 & & & \\
\hline $7-10$ points & 2.53 & 1.12 & 5.67 & 0.024 \\
\hline \multicolumn{5}{|c|}{ COVID-19 epidemiological survey: confirmed past infection } \\
\hline No & 1 & & & \\
\hline Yes & 0.21 & 0.05 & 0.91 & 0.038 \\
\hline _cons & 115.0 & 1.77 & 7455.3 & 0.026 \\
\hline
\end{tabular}

$T K R$ total knee replacement; $R K R$ replacement knee replacement; $N R S$ numeric rating scale

Model adjustment: $\operatorname{LR} \chi^{2}(5)=21.67 ; p=0.001$; Pseudo $R^{2}=0.1224$; $n=156$ undergo or refuse surgery (Table 5). Patients from the RKR waiting list were equally likely to undergo the surgery than patients from the TKR waiting list (OR 1.13; SE 0.78; $p=0.856$, n.s.); for each increase in a year of age, patients were $6 \%$ less likely to undergo surgery (OR $0.94 ; p=0.046$ ); patients with 7-10 points in the perceived pain NRS were 2.5 times at more odds to undergo surgery than patients with less pain (OR 2.53; SE 1.04; $p=0.024)$; patients that were diagnosed with COVID-19 infection before being surveyed, were $79 \%$ less likely to undergo surgery than patients with no history of COVID-19 infection (OR 0.21; SE 3.6; $p=0.038$ ); finally, patients with an ASA classification of III were $55 \%$ less likely to undergo surgery than patients with less punctuation (OR 0.45; SE 0.19 ; but at $7 \%$ of significance, $p=0.066$ ) (Table 5).

The probability of patients to prefer early surgery (Fig. 1left) was compared to that of patients effectively undergoing surgery when scheduled (Fig. 1-right), according to the age and pain. In the younger group of patients (under 65) with a perceived pain over 7 points, the mean probability did not significantly change (paired $t$ test; $p=0.344$ ), from the patient preference (left) to actually undergoing the scheduled surgery (right). However, when the perceived pain was under 7 points, the mean probability did change from 69 to $85 \%$ (paired $t$ test; $p=0.002$ ). In the group of patients older than 65 years with a perceived pain over 7 points, the mean probability to schedule surgery significantly changed (paired $t$ test; $p=0.009$ ) from 73\% (when the preference was surveyed) to $80 \%$ (accepting surgery when it was effectively scheduled). When the perceived pain was lower than 7 points, the mean probability did change from 32 to
Fig. 1 Probability to re-schedule or undergo surgery depending on the age and the perceived pain, in patients from the knee replacement waiting list established before the 1 st wave of the COVID-19 pandemic. Left: Probability of re-scheduling based on preferences expressed in the telephone survey. Right: Probability of undergoing surgery based on patients effectively undergoing surgery after the end of surgical lockdown
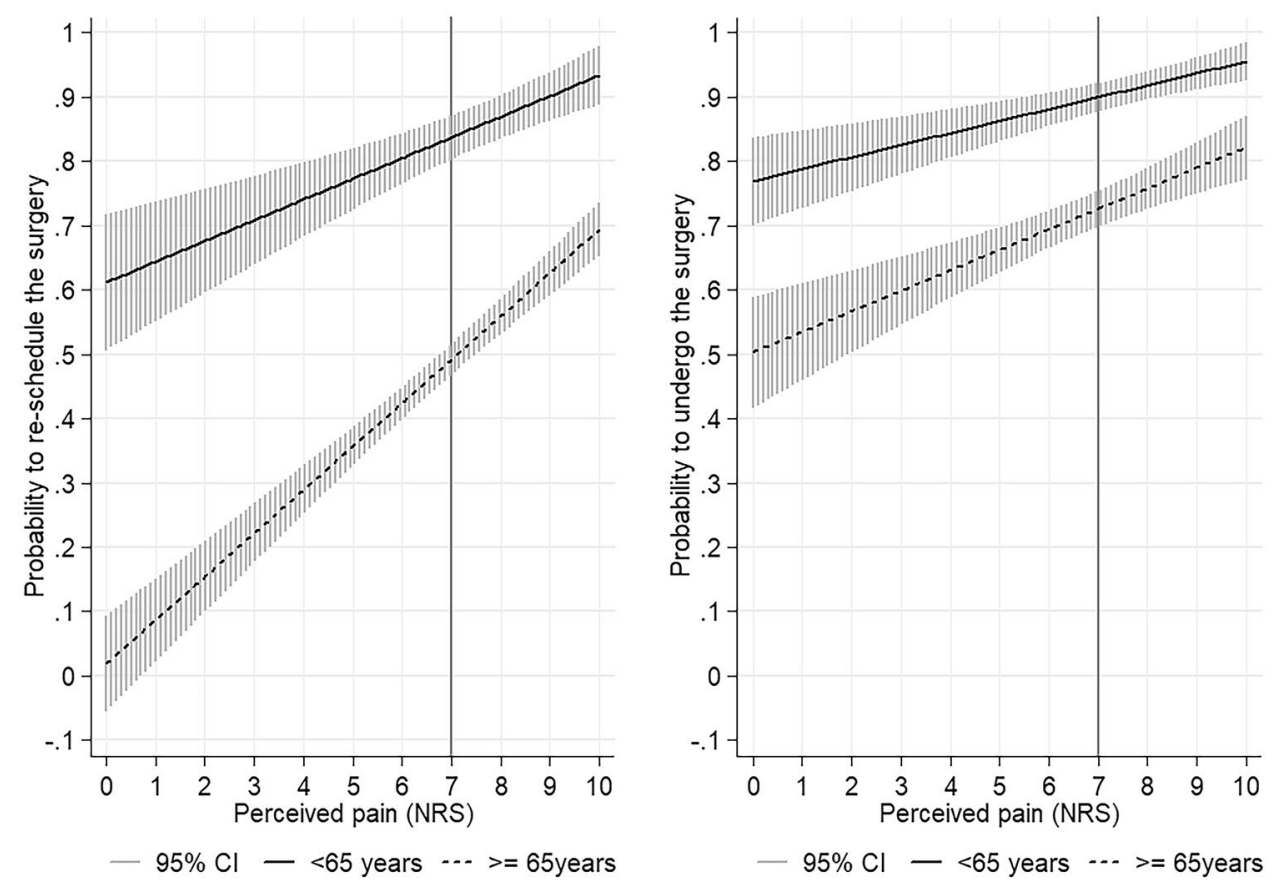
$63 \%$ (paired $t$ test; $p=0.000$ ) from preference to surgery acceptance.

\section{Discussion}

The main finding in this study is the fact that, despite the expected reluctancy to undergo the planned surgery for knee replacement after the 1st wave of the pandemic, severe pain prompts surgical acceptance. Also, younger patients were more prone to undergo surgery, regardless of the amount of pain and despite the pandemic.

The delays imposed by the pandemic lockdown (from 3 to 6 more months) are not without consequences. Other studies have proven that waiting lists produce a deterioration of the patients' health status [1]. This was reflected in our series particularly in young patients who proved eager to receive surgery despite the concerning situation of the pandemic.

The first wave of the COVID-19 pandemic severely affected elective procedures such as knee replacement in many hospitals. Surveys launched by European organizations [24] confirmed that in many countries, the elective joint replacement surgery underwent a lockdown. This implied serious clinical and functional deterioration of patient quality of life during and after the pandemic. Many patients refused to look for appropriate care in fear of the disease, and the uncertainty about the potential complications due to COVID-19 extensive occupation of hospitals. Recommendations issued after the first wave of the pandemic to reopen the elective surgery of total joint replacement, particularly knee replacement [14] and a standardized approach to treat patients in protective environments facilitated to regain trust in the healthcare system that performs elective procedures such as knee replacement. Still, the ultimate decision is at the patient's end, and understanding patient behaviours and needs became important to articulate specific responses that would foster this surgery.

In this context, we developed not only the description of what happened to our patients in the knee replacement waiting list at the time of the lockdown but more importantly, the comparison of different patient subgroups (primary versus revision knee replacement, higher risk patients, younger and older patients). Furthermore, the probability to accept scheduling knee replacement surgery was evaluated in this cohort of waiting list patients based on their answers about preferences in a telephone survey. This preference was then confronted with the real decision about scheduling surgery or refusing it when the procedure was available again.

The impact of the 1st wave of the COVID-19 not only affected seriously the population (in our hospital, the overall mortality of admitted patients was 20.7\%)[5], also the population was aware of the severity of the situation. While we investigated through the influence of stress and anxiety, as well as the proximity of COVID-19 to each patient, the potential role of fear to be admitted and undergo surgery at the hospital, only a previous diagnosis of COVID-19 in some patients influenced their actual acceptance to undergo the planned surgery, increasing the refusal. The PSS has been validated for elderly patients [9], but in our series, the stress values seemed to be lower than published in an elderly population (mean PSS $=17.1 \pm 7.6$ ) $[9,18]$, although our findings are consistent with females showing higher punctuation than males [3, 9]. We were not able to prove that high stress and anxiety levels influenced the preference of patients to schedule surgery, and more specifically, to effectively undergo surgery.

The rate of patients that initially preferred to delay surgery was high ( $47 \%$ for primary TKR), but when surgery was scheduled, up to $55 \%$ of patients initially postponing surgery finally accepted. Therefore, we developed predictive models about the variables that influenced surgery preferences for early scheduling, and surgery acceptance, and could conclude that age, pain, and previous diagnosis of COVID-19 were significant factors modifying the patient preferences to undergo surgery. Pain and age combined in a graph representation can be compared, as shown in Fig. 1, and different decision trends were identified.

The following limitations are acknowledged in our study. The number of patients could have been larger, and more information from subgroups could have been obtained. The subgroups are different in configuration and patient characteristics, and this is a limitation for comparisons. This limitation also affected the predictive model. The low sample size effect was observed in the high confidence intervals of age category, although the model is still valid and adjusted. Another limitation is that some variables could be affected by the social desirability bias, which means that patients may be inclined to present themselves with certain health behaviour in a more positive light [17]. It has been found that advancing age has been communicated associated with a decrease in self-reporting negative effects, and that telephone interviews would be prone to this type of bias [23]. This may explain the difference of almost 5 points in the stress scale with published data and the high percentage of patients that finally decided to undergo surgery when they first preferred to postpone it. Besides those mentioned limitations related to telephone questionnaires, sample, and subgroup size, our study provides an insight into the problems encountered to select patients to undergo the planned knee replacement during the pandemic.

\section{Conclusions}

Surgical timing preferences for knee replacement vary between patients older than 65 years (immediate surgery only when pain is intense) and younger patients (immediate 
surgery no matter the amount of pain). Even if COVID-19 severely stroke our population, the need for knee replacement stood in the young population and even in the aged population at risk for COVID when pain was important.

Supplementary Information The online version contains supplementary material available at https://doi.org/10.1007/s00167-021-06611-x.

Funding No funding was obtained for this study.

\section{Declarations}

Conflict of interest No conflicts of interest apply for any of the authors

Ethical approval Eligibility criteria included patients over 18 years old, in the surgical waiting list for knee replacement (whether TKR or RKR), able to give verbal consent to participate in the study, as approved by the Ethical Committee of La Paz University Hospital (Code: PI-4298, 31 July 2020).

\section{References}

1. Ackerman IN, Bennell KL, Osborne RH (2011) Decline in healthrelated quality of life reported by more than half of those waiting for joint replacement surgery: a prospective cohort study. BMC Musculoskelet Disord 12:108

2. Ahmad I, Konduru S (2007) Change in functional status of patients whilst awaiting prinmary total knee arthroplasty. Surgeon 5:266-267

3. Andreou E, Alexopoulos EC, Lionis C, Varvogli L, Gnardellis C, Chrousos GP et al (2011) Perceived Stress Scale: reliability and validity study in Greece. Int J Environ Res Public Health 8:3287-3298

4. Bird C (2013) Wait times increasing for hip and knee replacement. CMAJ 185:E325

5. Borobia AM, Carcas AJ, Arnalich F, Álvarez-Sala R, MonserratVillatoro J, Quintana M et al (2020) A cohort of patients with COVID-19 in a Major Teaching Hospital in Europe. J Clin Med. https://doi.org/10.3390/jcm9061733

6. British_Orthopaedic_Association. Re-starting non-urgent trauma and orthopaedic care: full guidance. 2020; version 1.1:https:// www.boa.ac.uk/resources/boa-guidance-for-restart---summary--final-pdf.html. Accessed 15/05/2020, 2020.

7. Comas M, Román R, Quintana JM, Castells X (2010) Unmet needs and waiting list prioritization for knee arthroplasty. Clin Orthop Relat Res 468:789-797

8. Desmeules F, Dionne CE, Belzile E, Bourbonnais R, Frémont $\mathrm{P}$ (2010) The burden of wait for knee replacement surgery: effects on pain, function and health-related quality of life at the time of surgery. Rheumatology (Oxford) 49:945-954

9. Ezzati A, Jiang J, Katz MJ, Sliwinski MJ, Zimmerman ME, Lipton RB (2014) Validation of the Perceived Stress Scale in a community sample of older adults. Int J Geriatr Psychiatry 29:645-652

10. Gutacker N, Siciliani L, Cookson R (2016) Waiting time prioritisation: evidence from England. Soc Sci Med 159:140-151

11. Jensen MP, Karoly P, O'Riordan EF, Bland F Jr, Burns RS (1989) The subjective experience of acute pain. An assessment of the utility of 10 indices. Clin J Pain 5:153-159

12. Jensen MP, Chen C, Brugger AM (2003) Interpretation of visual analog scale ratings and change scores: a reanalysis of two clinical trials of postoperative pain. J Pain 4:407-414
13. Kort NP, Barrena EG, Bédard M, Donell S, Epinette JA, Gomberg $B$ et al (2020) Recommendations for resuming elective hip and knee arthroplasty in the setting of the SARS-CoV-2 pandemic: the European Hip Society and European Knee Associates Survey of Members. Knee Surg Sports Traumatol Arthrosc 28:2723-2729

14. Kort NP, Barrena EG, Bédard M, Donell S, Epinette JA, Gomberg B et al (2020) Resuming elective hip and knee arthroplasty after the first phase of the SARS-CoV-2 pandemic: the European Hip Society and European Knee Associates recommendations. Knee Surg Sports Traumatol Arthrosc 28:2730-2746

15. Kort NP, Zagra L, Barrena EG, Tandogan RN, Thaler M, Berstock JR et al (2020) Resuming hip and knee arthroplasty after COVID19: ethical implications for wellbeing, safety and the economy. Hip Int 30:492-499

16. National Center for Immunization and Respiratory Diseases (NCIRD) DoVD. Groups at higher risk for severe illness. Coronavirus Disease 2019 (COVID-19) https://www.cdc.gov/coron avirus/2019-ncov/need-extra-precautions/groups-at-higher-risk. html. 17/06/2020.

17. Nolte S, Elsworth GR, Osborne RH (2013) Absence of social desirability bias in the evaluation of chronic disease self-management interventions. Health Qual Life Outcomes 11:114

18. Remor E (2006) Psychometric properties of a European Spanish version of the Perceived Stress Scale (PSS). Span J Psychol 9:86-93

19. Rossi R, Socci V, Talevi D, Mensi S, Niolu C, Pacitti F et al (2020) COVID-19 pandemic and lockdown measures impact on mental health among the general population in Italy. Front Psychiatry 11:790

20. Sanidad MD. Bienestar emocional. Estrategia de promoción de la salud y prevención en el SNS [Gov]. 2020; https://bemocion.sanid ad.gob.es/comoEstoy/autoevaluacionEstres/home.htm. Accessed 17/06/2020.

21. Scott CEH, MacDonald DJ, Howie CR (2019) 'Worse than death' and waiting for a joint arthroplasty. Bone Jt J 101-b:941-950

22. SEMPSP/AEC/SEIMC. Recomendaciones para la programación de cirugía en condiciones de seguridad durante el periodo de transición de la pandemia COVID-19. 2020; https://www.mscbs.gob. es/profesionales/saludPublica/ccayes/alertasActual/nCov-China/ documentos/200517-DOCUMENTO_CIRUGIA-FINAL_(2).pdf. Accessed 02 June 2020.

23. Soubelet A, Salthouse TA (2011) Influence of social desirability on age differences in self-reports of mood and personality. J Pers 79:741-762

24. Thaler M, Khosravi I, Hirschmann MT, Kort NP, Zagra L, Epinette JA et al (2020) Disruption of joint arthroplasty services in Europe during the COVID-19 pandemic: an online survey within the European Hip Society (EHS) and the European Knee Associates (EKA). Knee Surg Sports Traumatol Arthrosc 28:1712-1719

25. Viosca E, Martinez JL, Almagro PL, Gracia A, Gonzalez C (2005) Proposal and validation of a new functional ambulation classification scale for clinical use. Arch Phys Med Rehabil 86:1234-1238

26. Webb L (2020) COVID-19 lockdown: a perfect storm for older people's mental health. J Psychiatr Ment Health Nurs. https://doi. org/10.1111/jpm.12644

Publisher's Note Springer Nature remains neutral with regard to jurisdictional claims in published maps and institutional affiliations. 